Shiro Saka

\title{
Special issue on "Wood science and technology for mitigation of global warming"
}

Published online: October 28, 2009

Since the Industrial Revolution, our civilization has developed with the support of fossil fuels such as petroleum. However, the use of fossil fuels releases carbon dioxide $\left(\mathrm{CO}_{2}\right)$ and other greenhouse gases that cause global warming; recent indications point to the danger linked to weather anomalies, changes in ecosystems, and other radical transformations of the global environment. Moreover, there have been concerns since the middle of the twentieth century regarding depletion of fossil resources owing to their massive consumption, thus, it is feared, making it difficult for civilization to survive.

Under such circumstances, in recent years biofuels such as bioethanol and biodiesel, developed from biomass raw materials, have captured the world's attention as cleanenergy alternatives to petroleum. In Japan, as well, biofuels are regarded as one of the renewable "new energy" alternatives to petroleum, with the Kyoto Protocol for liquid biofuels allowing for 500 million liters (on a crude-oil basis) of liquid fuels to be used for transport by the year 2010. As part of this total, gasoline blended with bio-ETBE (ethyl tertiary butyl ether) went on sale in the greater Tokyo metropolitan area beginning on 27 April 2007, and the effective use of biomass resources is being advanced nationwide.

These biomass resources have been fixed and produced through the assimilation of $\mathrm{CO}_{2}$ in the atmosphere with water, using solar energy, so that the re-emission of its $\mathrm{CO}_{2}$ by the use of biomass such as burning is considered to be carbon-neutral because it will not increase net $\mathrm{CO}_{2}$ concentration in the atmosphere. However, for biomass resources to be used as biofuels, the following process is generally essential: collection of biomass $\rightarrow$ transportation $\rightarrow$ conversion $\rightarrow$ transportation $\rightarrow$ utilization. In order to obtain carbon-neutral biofuels from carbon-neutral biomass, such a process must be completed as economically and energyconservatively as possible. Therefore, we must minimize energy for transportation, conversion, and related factors. In addition, multistep usage of biomass wastes such as recycling is known to be important. Furthermore, efficient fixation of $\mathrm{CO}_{2}$ by afforestation as well as reforestation is of great importance to maintain sustainable ecosystems.

Based on such a basic concept of energy science, the Journal of Wood Science (JWS) is publishing this special issue of current research on wood science and technology for mitigation of global warming. Members of the Japan Wood Research Society as well as researchers abroad were encouraged to submit manuscripts for the special issue. Among the submitted and accepted manuscripts, the 10 most appropriate papers on this theme were selected by the Editorial Board of JWS. Papers not selected will be treated as original submissions for a future general issue.

Through the opportunity provided in this special issue, researchers expound the merits of wood in contributing to mitigation of global warming, leading to the efficient utilization of biofuels not only nationwide but also worldwide.

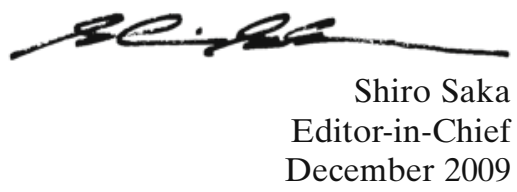

S. Saka $(\square)$

Department of Socio-Environmental Energy Science, Graduate School of Energy Science, Kyoto University, Yoshida-honmachi, Sakyo-ku, Kyoto 606-8501, Japan

Tel. +81-75-753-4738; Fax +81-75-753-4738

e-mail: saka@energy.kyoto-u.ac.jp 